\title{
Complexity of Clausal Constraints Over Chains*
}

\author{
Nadia Creignou ${ }^{\dagger}$ \\ LIF (CNRS, UMR 6166) \\ Univ. de la Méditerranée \\ 13288 Marseille cedex 9, France \\ Andrei Krokhin ${ }^{\S}$ \\ Department of Computer Science \\ University of Durham \\ Durham, DH1 3LE, United Kingdom
}

\author{
Miki Hermann $\ddagger$ \\ LIX (CNRS, UMR 7161) \\ École Polytechnique \\ 91128 Palaiseau cedex, France \\ Gernot Salzer $₫$ \\ Technische Universität Wien \\ Favoritenstraße 9-11 \\ A-1040 Wien, Austria
}

\begin{abstract}
We investigate the complexity of the satisfiability problem of constraints over finite totally ordered domains. In our context, a clausal constraint is a disjunction of inequalities of the form $x \geq d$ and $x \leq d$. We classify the complexity of constraints based on clausal patterns. A pattern abstracts away from variables and contains only information about the domain elements and the type of inequalities occurring in a constraint. Every finite set of patterns gives rise to a (clausal) constraint satisfaction problem in which all constraints in instances must have an allowed pattern. We prove that every such problem is either polynomially decidable or NPcomplete, and give a polynomial-time algorithm for recognizing the tractable cases. Some of these tractable cases are new and have not been previously identified in the literature.
\end{abstract}

\section{Introduction}

Research in complexity of constraint satisfaction problems (CSP) gained a considerable interest in the recent years. The first complete classification by means of a dichotomy theorem for Boolean CSP of Schaefer [Sch78] has been followed in the last decade by an intensive effort to extend his result to larger domains. Feder and Vardi [FV98] conjectured that a dichotomy theorem holds for every finite domain. This conjecture has been partially confirmed in 2002 when Bulatov [Bul02] proved a dichotomy theorem for the 3-element domain. The problem remains open for domains of higher cardinality. Confronted with the difficulty of the main goal, researchers started to investigate CSP problems with additional structure, like list constraints or conservative constraints [Bul03]. The effort has been pursued along several lines: one of them applies methods from universal algebra [BJK05, Bul02, Bul03], the other one is oriented towards graph theoretic methods [FV98, HN04], and there is also a finite model theory approach [Dal02, FV98, KV00].

\footnotetext{
${ }^{*}$ The work has been supported by ÉGIDE 06606ZF and ÖAD Amadeus 18/2004.

${ }^{\dagger}$ email: Nadia.Creignou@lif.univ-mrs.fr

‡email: Miki.Hermann@lix.polytechnique.fr

§email: Andrei.Krokhin@durham.ac.uk

ฯemail: salzer@logic .at
} 
Dichotomy results are important in computational complexity. Ladner proved in [Lad75] that there exists an infinite hierarchy between the class $\mathrm{P}$ of polynomial-time decidable problems and $\mathrm{NP}$-complete problems, provided that $\mathrm{P} \neq \mathrm{NP}$. On the other hand, a dichotomy theorem states that a considered constraint satisfaction problem is either polynomial-time decidable or NP-complete, depending on a parameter usually presented in the form of a finite set of relations.. This means that dichotomy results are not at all obvious and should constitute an exception under the hypothesis that $\mathrm{P} \neq \mathrm{NP}$.

In this paper we consider constraint satisfaction problems inspired by many-valued logic. In fact, we take for basis of our research the regular signed logic [Häh01], where the underlying finite domain is totally ordered, i.e., is a chain. This logic provides us with the concept of literal, clause, and formula, which extend naturally from the Boolean domain. Moreover, the well-known polynomialtime decidable satisfiability cases, namely Horn, dual Horn, bijunctive, 0- and 1-valid preserve their good complexity properties. The cornerstone of our approach is the concept of clausal pattern, which is an abstraction of all clauses of certain type. These patterns correspond, roughly speaking, to constraints in the algebraic approach. Finite sets of patterns constitute clausal languages upon which we construct formulas, whose satisfiability are at the heart of our CSP problems. Naturally, these CSP problems are parametrized by clausal languages. Different clausal languages lead to CSP problems of different complexity. First, we get rid of the redundant values in the domain, and thus concentrate on clausal languages closed under taking subpatterns and containing all unary clauses. Next, to be able to compare clausal languages and subsequently the complexity of the induced CSP problems, we build 3-saturations by means of resolution and subpatterns. Roughly speaking, the 3 -saturation of a clausal language $L$ gives all patterns of length at most 3 that can be "implemented" by $L$, thus measuring the expressive power of $L$.

There is no direct relationship between classical constraint satisfaction problems based on relations and our CSPs based on clausal languages. Of course, for each clausal CSP there exists a classical CSP with the same expressive power, but there are relation-based CSPs with no equivalent clausal CSP. This is due to the fact that a clausal pattern is a prototype of a clause in regular manyvalued logic, contrary to a relation upon a domain. A pattern gives rise to a particular relation composed of vectors satisfying the intermediate clause. Indeed, each relation is a set of satisfying assignments of a conjunction of clauses, as it was proved in [GHSZ04], but there are relations that cannot be represented by a single clause.

We first derive the exact condition for NP-complete CSP problems, followed by a careful and exhaustive analysis on the patterns occurring in the saturation, which provide us with the polynomialtime decidable cases. We obtain a dichotomy theorem for any cardinality of the finite totally ordered domain, where the previously known four polynomial-time decidable cases keep their complexity. Moreover, we discover new polynomial-time decidable cases, which are absent from Boolean CSP problems. We present an algorithm computing a satisfying assignment of a formula, if it exists, by a unified approach for all polynomial-time decidable cases. Hence, we obtain a complete classification of many-valued logics-based CSP problems over finite totally ordered domains.

\section{Preliminaries}

Let $D$ be a finite chain, say $D=\{0,1, \ldots, n-1\}$ with the total order $0<1<\cdots<n-1$, called a domain, and let $V$ be a set of variables. For $x \in V$ and $d \in D$, the inequalities $x \geq d$ and $x \leq d$ are called positive and negative literals, respectively. A clause is a disjunction of literals. As usually, 
an interval $[a, b]$ denotes the subset of values $\{x \in D \mid a \leq x \leq b\}$.

A clausal pattern, or pattern for short, is a multiset ${ }^{1}$ of the form

$$
P=\left(+a_{1}, \ldots,+a_{p},-b_{1}, \ldots,-b_{q}\right),
$$

where $p, q \in \mathbb{N}$ and $a_{i}, b_{i}$ are values from the domain $D$, for all $i$. The $+a_{i}$ 's are called positive literals, the $-b_{i}$ 's negative literals. The sum $p+q$, also denoted by $|P|$, is the length of the pattern. Denote by $P[l]$ the number of occurrences of the literal $l$ in the pattern $P$. If $P[l] \leq Q[l]$ holds for all literals $l$ then $P$ is called a subpattern of $Q$. Note that there are at least $|Q|$ and at most $2^{|Q|}$ possible subpatterns of $Q$, depending on the repeated occurrences of literals, including also $Q$ and the empty subpatterns. A clausal language $L$ is a finite set of clausal patterns, with arities not necessarily equal. We denote by $P^{+}$and $P^{-}$the positive and negative parts, respectively, of the pattern $P$, i.e., $P^{+}=\left(+a_{1}, \ldots,+a_{p}\right)$ and $P^{-}=\left(-b_{1}, \ldots,-b_{q}\right)$. We denote by $\min \left(P^{+}\right)=\min \left\{a_{1}, \ldots, a_{p}\right\}$ and $\max \left(P^{-}\right)=\max \left\{b_{1}, \ldots, b_{q}\right\}$ the minimum and maximum values of the parts $P^{+}$and $P^{-}$, respectively. The values $\min \left(P^{+}\right)$and $\max \left(P^{-}\right)$are undefined for empty parts $P^{+}$and $P^{-}$. We denote by $\operatorname{pos}_{\ell}(L)=\left\{P \in L\left|P=P^{+},\right| P \mid=\ell\right\}$ and $\operatorname{neg}_{\ell}(L)=\left\{N \in L\left|N=N^{-},\right| N \mid=\ell\right\}$ the set of all positive and negative patterns of length $\ell$ in $L$, respectively.

Given a clausal language $L$ and a countably infinite set of variables $V$, an $L$-clause is a pair $(P, \vec{x})$, where $P \in L$ is a pattern and $\vec{x}$ is a finite vector of not necessarily distinct variables from $V$, such that $|P|=|\vec{x}|$. A pair $(P, \vec{x})$ with a pattern $P=\left(+a_{1}, \ldots,+a_{p}, \ldots,-b_{1}, \ldots,-b_{q}\right)$ and variables $\vec{x}=\left(x_{1}, \ldots, x_{p+q}\right)$ represents the clause $c_{P}=\left(x_{1} \geq a_{1} \vee \cdots \vee x_{p} \geq a_{p} \vee x_{p+1} \leq b_{1} \vee \cdots \vee x_{p+q} \leq b_{q}\right)$. We will use the more conventional notation $P(\vec{x})$ instead of $(P, \vec{x})$. An $L$-formula, or formula for short, is a conjunction of a finite number of $L$-clauses. We denote by $\varphi\left(x_{1}, \ldots, x_{k}\right)$ that the variables $x_{1}, \ldots, x_{k}$ occur in $\varphi$, fixing also implicitly their sequence.

An assignment is a mapping $I: V \rightarrow D$ assigning a domain element $I(x)$ to each variable $x \in V$. The satisfaction relation $I \models \varphi$ is defined as follows:

- $I \models$ true and $I \not \models$ false;

- $I \models x \leq d$ if $I(x) \leq d$;

- $I \models x \geq d$ if $I(x) \geq d$;

- $I \models \varphi \wedge \psi$ if $I \models \varphi$ and $I \models \psi$;

- $I \models \varphi \vee \psi$ if $I \models \varphi$ or $I \models \psi$.

If $I \models \varphi$ holds, we say that $I$ satisfies $\varphi$.

The well-known resolution rule from Boolean logic has a straightforward extension to regular many-valued logic based on a totally ordered domain. Consider two clauses $C_{1}=(C \vee x \geq a)$ and $C_{2}=\left(x \leq b \vee C^{\prime}\right)$ with a variable $x$, the subclauses $C$ and $C^{\prime}$, and two values $a, b \in D$ satisfying the condition $a>b$. Then $C \vee C^{\prime}$ is called a resolvent of the parent clauses $C_{1}$ and $C_{2}$. Resolvents are logical consequences of their parent clauses, i.e., if an assignment satisfies $C_{1}$ and $C_{2}$, then it also satisfies $C \vee C^{\prime}$ [BFS01].

It can be easily seen that the literals +0 and $-(n-1)$ are superfluous since the inequalities $x \geq 0$ and $x \leq n-1$ are always satisfied. We call these literals trivial, contrary to the non-trivial

\footnotetext{
${ }^{1} \mathrm{~A}$ multiset (also called bag) is a set with repetitions.
} 
literals $+1, \ldots,+(n-1)$ and $-0, \ldots,-(n-2)$. Without loss of generality, it is sufficient to only consider patterns and clausal languages with non-trivial literals.

A clausal pattern $P=\left(+a_{1}, \ldots,+a_{p},-b_{1}, \ldots,-b_{q}\right)$ is said to be

- Horn if $p \leq 1$,

- dual Horn if $q \leq 1$,

- bijunctive if $p+q \leq 2$ and binary if $p+q=2$,

- $d$-valid if $P(\vec{x})$ is satisfied by the constant assignment $I(x)=d$ for all $x \in V$,

- positive if $p>0$ and $q=0$ (i.e., $P=P^{+}$),

- negative if $p=0$ and $q>0$ (i.e., $P=P^{-}$),

- monotone if it is positive or negative,

- mixed if $p>0$ and $q>0$.

A clausal language $L$ is Horn, dual Horn, bijunctive, or $d$-valid, respectively, if every pattern in $L$ has the corresponding property.

A relation $R$ of arity $k$ over a finite domain $D$ is a subset $R \subseteq D^{k}$. A vector $m \in R$ is denoted by $m=(m[1], \ldots, m[k])$, where $m[i]$ is the value of $m$ at the $i$-th coordinate. Each $L$-formula $\varphi$ gives rise to a corresponding relation

$$
\operatorname{Sol}\left(\varphi\left(x_{1}, \ldots, x_{k}\right)\right)=\left\{\left(I\left(x_{1}\right), \ldots, I\left(x_{k}\right)\right) \mid I \models \varphi\right\}
$$

produced by the set of assignments $I$ satisfying $\varphi$.

Let $R \subseteq D^{k}$ be a relation of arity $k$ over the domain $D$ and $f: D \rightarrow D$ be a unary function. The image of the relation $R$ under the function $f$ is the set of vectors

$$
f(R)=\{(f(m[1]), \ldots, f(m[k])) \mid m \in R\}
$$

of arity $k$. We say that the relation $R$ is closed under $f$ if the inclusion $f(R) \subseteq R$ holds.

In the sequel we need the image of clausal patterns under unary functions. Let $P$ be a clausal pattern of length $k$ over the domain $D$ and $f: D \rightarrow D$ be a unary function. The image of the pattern $P$ under the function $f$ is the relation

$$
f(P)=\left\{f(m) \mid m \in \operatorname{Sol}\left(P\left(x_{1}, \ldots, x_{k}\right)\right)\right\} .
$$

We say that a pattern $P$ is closed under $f$ if the inclusion $f(P) \subseteq \operatorname{Sol}\left(P\left(x_{1}, \ldots, x_{k}\right)\right)$ holds. In this case, $f$ is called an endomorphism (or unary polymorphism) of $P$. A unary function $f$ is called an endomorphism of a clausal language $L$ if the inclusion $f(P) \subseteq \operatorname{Sol}\left(P\left(x_{1}, \ldots, x_{k}\right)\right)$ holds for each $P \in L$.

Let $f: D \rightarrow D$ be a unary function defined on the domain $D$. We denote the range of $f$ by $f(D)$ and the $k$-fold composition of $f$ by $f^{k}$ (defined recursively as $f^{1}=f$ and $f^{k+1}=f^{k} \circ f$ ). 


\section{Constraint Satisfaction Problems}

Given a clausal language $L$, the constraint satisfaction problem over $L$ is defined as follows.

Problem: $\operatorname{Csp}(V, D, L)$

Input: An $L$-formula $\varphi$ over a finite totally ordered domain $D$ and variables $V$.

Question: Is $\varphi$ satisfiable?

If the domain $D$ and variables $V$ are implicitly clear, we write $\operatorname{CSP}(L)$ instead of $\operatorname{CsP}(V, D, L)$.

In this section we will show that, in order to classify the complexity of problems $\operatorname{CsP}(L)$, it is sufficient to do this for languages which contain all unary patterns and are closed under taking subpatterns.

Lemma 1 Let $L$ be a clausal language such that for some $a \in\{1, \ldots, n-1\}$ the positive literal $+a$ (or for some $a \in\{0, \ldots, n-2\}$ the negative literal $-a$ ) does not occur in any pattern in $L$. Then there exists a clausal language $L^{\prime}$ over the domain $D^{\prime}=D \backslash\{a\}$ such that $\operatorname{CSP}(V, D, L)$ and $\operatorname{CSP}\left(V, D^{\prime}, L^{\prime}\right)$ are polynomial-time equivalent.

Proof: We only consider the case of a positive literal $+a$ not occurring in $L$; the other case is dual. For every pattern $P \in L$, let $P^{\prime}$ be a pattern over $D^{\prime}$ obtained from $P$ by replacing each occurrence of $-a$ by $-(a-1)$, and let $L^{\prime}=\left\{P^{\prime} \mid P \in L\right\}$. We claim that $\operatorname{Csp}(V, D, L)$ and $\operatorname{csp}\left(V, D^{\prime}, L^{\prime}\right)$ are polynomial-time equivalent.

Given a formula $\varphi$ as an instance of $\operatorname{CSP}(V, D, L)$, construct the new formula $\varphi^{\prime}$ from $\varphi$ by replacing every literal of the form $x \leq a$ by $x \leq a-1$. Obviously $\varphi^{\prime}$ is an instance of $\operatorname{CSP}\left(V, D^{\prime}, L^{\prime}\right)$. Conversely, given an instance $\varphi^{\prime}$ of $\operatorname{CSP}\left(V, D^{\prime}, L^{\prime}\right)$, construct $\varphi$ from $\varphi^{\prime}$ by replacing literals of the form $x \leq a-1$ by $x \leq a$ in those clauses, which do not correspond to any pattern in $L$. The modified clauses now correspond to patterns in $L$, i.e., $\varphi$ is an instance of $\operatorname{CsP}(V, D, L)$.

We show that $\varphi$ and $\varphi^{\prime}$ are logically equivalent. Every assignment satisfying $\varphi^{\prime}$ also satisfies $\varphi$ since $x \leq a-1$ implies $x \leq a$. Conversely, let $I$ be an assignment satisfying $\varphi$, and let $I^{\prime}$ be the assignment defined as $I^{\prime}(x)=a-1$ if $I(x)=a$, and $I^{\prime}(x)=I(x)$ otherwise. By a case analysis it is straightforward to verify that for every literal in $\varphi$ satisfied by $I$, the corresponding literal in $\varphi^{\prime}$ is satisfied by $I^{\prime}$ (bearing in mind that $\varphi$ does not contain literals of the form $x \geq a$ ).

Remark 2 It follows from the definitions that if $f$ is an endomorphism of $L$ and $I$ is a satisfying assignment to an instance of $\operatorname{CSP}(L)$ then $f \circ I$ also satisfies the instance. In particular, every satisfiable instance of $\operatorname{CsP}(L)$ has a solution using only values from $f(D)$.

Lemma 3 Let $L$ be a clausal language over a domain $D$ that has an endomorphism $f$ which is not a permutation. Then there exists a clausal language $L^{\prime}$ on a smaller domain $D^{\prime}$ such that $\operatorname{CsP}(V, D, L)$ and $\operatorname{CsP}\left(V, D^{\prime}, L^{\prime}\right)$ are polynomial-time equivalent.

Proof: It is easy to see that for any $k$ the iterated function $f^{k}$ is also an endomorphism and that for some $k$ the mapping $f^{k}$ acts identically on its range, i.e., that $f^{k}(a)=a$ holds for every $a \in f^{k}(D)$. Without loss of generality, we may assume that already the unary function $f$ has this property. Since $f$ is not a permutation, we can find an element $b \in D$ which is not in the range of $f$, i.e., such that $b \notin f(D)$. Let $f(b)=a$ for some $a \in f(D)$. We have either $a<b$ or $a>b$. We perform the proof only for the case $a<b$, since the other one is similar. 
Let $D=\{0, \ldots, n-1\}$ be the domain. We need a case analysis corresponding to the position of $b$ in $D$. If $b=n-1$ then every satisfiable instance of $\operatorname{CsP}(V, D, L)$ has a solution over $\{0, \ldots, n-2\}$ according to Remark 2. So we can simply remove $n-1$ from $D$ and all occurrences of $n-1$ from the patterns in $L$. Clearly this leads to an equivalent problem over a smaller domain.

Let $b<n-1$. For every pattern $P \in L$, let $P^{\prime}$ be a pattern obtained from $P$ by replacing each literal $+b$ (if there are any) by $+(b+1)$. Set $L^{\prime}=\left\{P^{\prime} \mid P \in L\right\}$ and $D^{\prime}=D \backslash\{b\}$. We claim that $\operatorname{CSP}(V, D, L)$ and $\operatorname{CSP}\left(V, D^{\prime}, L^{\prime}\right)$ are polynomial-time equivalent. Since $f$ is also an endomorphism of $L^{\prime}$, following Remark 2 every satisfiable instance of $\operatorname{CsP}\left(V, D^{\prime}, L^{\prime}\right)$ has a satisfying assignment $I: V \rightarrow f(D)$ using only values from the range of $f$. Clearly, changing all literals of the form $x \geq b$ to $x \geq b+1$ does not affect the satisfiability of instances of $\operatorname{CsP}(V, D, L)$.

By Lemmas 1 and 3, in order to classify the complexity of problems $\operatorname{CSP}(L)$, it is sufficient to consider only clausal languages $L$ whose all endomorphisms are permutations and such that each non-trivial literal is present in some pattern in $L$. To further restrict the class of clausal languages, we need to temporarily extend the type of constraints under consideration by allowing not only constraints given by patterns from $L$, but also constant constraints, i.e., constraints of the form $x=a$ where $x$ is a variable and $a$ is an element from $D$. For a clausal language $L, \operatorname{let}^{\operatorname{CSP}_{c}}(L)$ denote the extended problem $\operatorname{CSP}(L)$ in which arbitrary constant constraints are also allowed in instances. Note that we can express the constraint $x=a$ as a conjunction of two unary clauses $(x \leq a) \wedge(x \geq a)$.

Proposition 4 ([BJK99, BJK05]) $\operatorname{CSP}(L)$ and $\operatorname{CSP}_{c}(L)$ are polynomial-time equivalent whenever all endomorphisms of a clausal language $L$ are permutations.

Proof: Corollary 4.8 in [BJK05] or Theorem 3.7 in [BJK99].

Definition 5 We call a clausal language $L$ SU-closed, if it is closed under taking subpatterns and it contains all non-trivial unary patterns, i.e., if it satisfies the following conditions:

1. If $P \in L$ and $P^{\prime}$ is a subpattern of $P$ then $P^{\prime} \in L$.

2. $(+d) \in L$ for all $d \in\{1, \ldots, n-1\}$.

3. $(-d) \in L$ for all $d \in\{0, \ldots, n-2\}$.

We need first to determine the maximal possible cardinality of SU-closed clausal languages, what is done be means of the following combinatorial lemma.

Lemma 6 There are at most $(\ell+1)^{2|D|-2}$ different patterns of length at most $\ell$ over a domain $D$.

Proof: Let $D=\{0, \ldots, n-1\}$. Every pattern $P$ can be uniquely described by the characteristic vector $\left(k_{+1}, \ldots, k_{+(n-1)}, k_{-0}, \ldots, k_{-(n-2)}\right)$, where $k_{l}$ denotes the number of occurrences of literal $l$ in pattern $P$. We have $0 \leq k_{l} \leq \ell$ since $P$ is of length at most $\ell$. The vector is of size $2(n-1)=2|D|-2$. Hence there are at most $(\ell+1)^{2|D|-2}$ different characteristic vectors and thus also patterns.

Proposition 7 For any clausal language $L$ over a fixed domain $D$ there exists an $S U$-closed clausal language $L^{\prime}$, such that $\operatorname{csP}(L)$ and $\operatorname{CsP}\left(L^{\prime}\right)$ are polynomial-time equivalent and, moreover, $L^{\prime}$ can be constructed from $L$ in polynomial time. 
Proof: To be able to apply Proposition 4, we describe an algorithm looking for non-surjective endomorphisms of the clausal language $L$. Using these endomorphisms, we apply Lemmas 1 and 3 to transform the language $L$ to another one having only permutative endomorphisms. Finally, using Proposition 4, we prove the polynomial-time equivalence between $\operatorname{CsP}(L)$ and $\operatorname{CsP}\left(L^{\prime}\right)$, where $L^{\prime}$ is the SU-closed clausal language corresponding to $L$.

First, we describe a polynomial-time algorithm which finds a non-surjective endomorphism of $L$ or shows that such an endomorphism does not exist. Let $P=\left(+a_{1}, \ldots,+a_{p},-b_{1}, \ldots,-b_{q}\right)$ be a pattern in $L$ and $R_{P}=\operatorname{Sol}\left(P\left(x_{1}, \ldots, x_{p+q}\right)\right)$ be the corresponding relation on $D$. By definition, a unary function $f$ on $D$ is not an endomorphism of $R_{P}$ if and only if there exists a vector $m=\left(m_{1}, \ldots, m_{p}, m_{1}^{\prime}, \ldots, m_{q}^{\prime}\right) \in R_{P}$, such that $f(m) \notin R_{P}$, i.e., if and only if there exists a vector $m=\left(m_{1}, \ldots, m_{p}, m_{1}^{\prime}, \ldots, m_{q}^{\prime}\right)$ such that the following conditions hold:

1. There exists an $i$, such that $i \in\{1, \ldots, p\}$ and $m_{i} \geq a_{i}$ or $i \in\{1, \ldots, q\}$ and $m_{i}^{\prime} \leq b_{i}$.

2. For all $j \in\{1, \ldots, p\}$ we have $f\left(m_{j}\right)<a_{j}$.

3. For all $j \in\{1, \ldots, q\}$ we have $f\left(m_{j}^{\prime}\right)>b_{j}$.

This can be checked simply by computing, for any fixed endomorphism $f$ and pattern $P$, all sets $\left\{m_{j} \mid f\left(m_{j}\right)<a_{j}\right\}$ and $\left\{m_{j}^{\prime} \mid f\left(m_{j}^{\prime}\right)>b_{j}\right\}$, which requires $|D| \times|P|$ time, following by a check whether one of these sets contains an element satisfying Condition 1. Since $|D|$ is a constant, for any given $f$ the above conditions can be verified in polynomial time. Since $D$ is fixed, the number of possible unary functions on $D$ is a constant.

Using the above algorithm, we can detect non-surjective endomorphisms of $L$. If $f$ is such an endomorphism and $b$ is an element absent from the range of $f$ then we follow the (linear-time) procedures from the proofs of Lemmas 1 and 3 to obtain an equivalent language over domain $D \backslash\{b\}$. We perform these steps until all endomorphisms of the obtained language are permutations, i.e., at most $|D|$ times.

By Lemmas 1 and 3, we may assume that all endomorphisms of $L$ are permutations and that each non-trivial literal is present in some pattern in $L$. We will show that the language $L^{\prime}$ obtained from $L$ by adding all unary patterns together with all subpatterns of the patterns in $L$ is the required language. Note that every unary pattern is a subpattern of some pattern in $L$ and that there are no more than $(\ell+1)^{2|D|-2}$ patterns in $L^{\prime}$, according to Lemma 6 , where $\ell$ is the maximal length of patterns in the considered clausal language $L$. Since the domain $D$ is fixed, i.e., the cardinality $|D|$ is a constant, the cardinality of $L^{\prime}$ is polynomial in the size of $L$. Hence, the language $L^{\prime}$ can be produced in polynomial time with respect to the size of $L$.

We will show that $\operatorname{CSP}(L)$ and $\operatorname{CSP}_{c}\left(L^{\prime}\right)$ are polynomial-time equivalent. This, together with obvious inclusions $\operatorname{CSP}(L) \subseteq \operatorname{CSP}\left(L^{\prime}\right) \subseteq \operatorname{CSP}_{c}\left(L^{\prime}\right)$, will prove the proposition.

As we noticed in Proposition 4, the problem $\operatorname{CSP}(L)$ is polynomial-time equivalent to $\operatorname{CSP}_{c}(L)$. It is easy to show that $\operatorname{CSP}_{c}(L)$ and $\operatorname{CSP}_{c}\left(L^{\prime}\right)$ are polynomial-time equivalent. Assume that $P=$ $\left(+a_{1},+a_{2}, \ldots,+a_{p},-b_{1}, \ldots,-b_{q}\right) \in L$ and $Q=\left(+a_{2}, \ldots,+a_{p},-b_{1}, \ldots,-b_{q}\right)$. Clearly the clause $\left(x_{2} \geq a_{2} \vee \ldots \vee x_{p} \geq a_{p} \vee x_{p+1} \leq b_{1} \vee \ldots \vee x_{p+q} \leq b_{q}\right)$ is obtained by resolution in many-valued logic from the clauses $x_{1} \leq 0$ and $\left(x_{1} \geq a_{1} \vee x_{2} \geq a_{2} \vee \ldots \vee x_{p} \geq a_{p} \vee x_{p+1} \leq b_{1} \vee \ldots \vee x_{p+q} \leq b_{q}\right)$. The clause $x_{1} \leq 0$ ensures that the variable $x$ is assigned the constant 0 in the latter clause and therefore it plays the role of the constant substitution $x_{1}=0$. Similarly, all patterns obtained from patterns of $L$ by removing one literal can be added to $L$, and the new problem will be equivalent 
to $\operatorname{CSP}_{c}(L)$. Continuing this procedure, we will eventually obtain that $\operatorname{CSP}_{c}(L)$ and $\operatorname{CSP}_{c}\left(L^{\prime}\right)$ are polynomial-time equivalent, which implies that $\operatorname{CSP}(L)$ and $\operatorname{CSP}_{c}\left(L^{\prime}\right)$ are equivalent.

Remark 8 Note that we always consider the domain $D$ to be fixed. If the domain $D$ were a part of the input, the SU-closed language $L^{\prime}$ would be exponentially larger that the corresponding clausal language $L$. Nevertheless, even in this case the problems $\operatorname{csp}(L)$ and $\operatorname{csp}\left(L^{\prime}\right)$ would be polynomial-time equivalent.

According to Lemma 3, we can get rid of redundant values in the domain. According to Proposition 7, we can restrict the $\operatorname{CSP}(L)$ problems to the case where the clausal language $L$ is closed under taking subpatterns and it contains all unary patterns. Therefore we consider only SU-closed clausal languages $L$ in the sequel.

\section{Complexity of CSP Problems}

Some polynomial cases have already been identified in the literature before without the use of clausal patterns and languages. The satisfiability problems for $L$-formulas built from Horn, dual Horn, and bijunctive clausal languages, respectively, were studied in the framework of many-valued logics [Häh01] as well as in the one of constraints [CJJK00, JC95].

Proposition 9 ([CJJK00, Häh01, JC95]) $\operatorname{CSP}(L)$ for a Horn, dual Horn, or bijunctive clausal language $L$ is decidable in polynomial time.

This result does not prove all polynomial-time decidable cases of $\operatorname{CsP}(L)$, but it shows that three polynomial-time decidable classes of Boolean constraint satisfaction problems extend to finite totally ordered domains, provided that we use the clausal patterns paradigm.

As in the Boolean case, it turns out that it is sufficient to examine the patterns of length at most 3 that can be obtained from $L$ (or "implemented" by $L$, see [CKS01]). For this reason we introduce the notion of 3-saturation of a clausal language which is based on the concept of pattern resolution. Let $P=\left(v_{1}, \ldots, v_{p},+a\right)$ and $Q=\left(-b, v_{1}^{\prime}, \ldots, v_{q}^{\prime}\right)$ be two patterns, such that $P$ contains a positive literal $+a$ and $Q$ a negative literal $-b$, satisfying $b<a$. Then the pattern $R=\left(v_{1}, \ldots, v_{p}, v_{1}^{\prime}, \ldots, v_{q}^{\prime}\right)$ is called a resolvent of $P$ and $Q$. We say that $R$ is obtained from $P$ and $Q$ by pattern resolution.

Definition 10 Let $L$ be an SU-closed clausal language, i.e. a clausal language closed under taking subpatterns and containing all unary patterns. The 3-saturation of $L$, denoted by $\widehat{L}$, contains all patterns that can be constructed inductively from $L$ by the following rules:

1. If $P \in L$ and $|P| \leq 3$, then $P \in \widehat{L}$ (introduction).

2. If $P$ and $Q$ are patterns in $\widehat{L}$ such that $|P|+|Q| \leq 5$, then all resolvents of $P$ and $Q$ are in $\widehat{L}$ (restricted resolution).

Note that due to the restriction, only resolvents with a length at most 3 are considered in the second condition. 
Remark 11 Observe that $\widehat{L}$ is an SU-closed language and that it can be computed from the already SU-closed language $L$ in linear time. This requires first to search $L$ for patterns of length smaller or equal to 3, according to Step 1, what can be done in linear time with respect to the size of $L$. Then the 3 -saturation $\widehat{L}$ is produced in constant time according to Step 2 .

Proposition $12 \operatorname{CSP}(\widehat{L})$ is reducible to $\operatorname{CSP}(L)$ in polynomial time.

Proof: For each formula $\varphi(\vec{x})$ over $\widehat{L}$ there exists a formula $\varphi^{\prime}(\vec{x}, \vec{y})$ over $L$, such that $\varphi^{\prime}$ can be obtained from $\varphi$ by means of resolution in polynomial time. It follows from resolution for many-valued regular logic (and is easy to see) that the formula $\varphi$ is satisfiable if and only if $\varphi^{\prime}$ is.

We need to analyze carefully which parts of the $\operatorname{CSP}(L)$ problem are polynomial-time decidable and which are NP-complete. To this end we need the concept of disjoint PN-pair.

Definition 13 We call two patterns $P$ and $N$ a $\mathbf{P N}$-pair if $P$ is positive $\left(P=P^{+}\right), N$ is negative $\left(N=N^{-}\right)$, one of them has length 2 , and the other has length 3. A PN-pair $(P, N)$ is called disjoint when $\max (N)<\min (P)$ holds, otherwise it is overlapping.

We will perform a reduction from the following well-known problem, which is also known as a constraint satisfaction problem over the set of Boolean relations $\left\{\right.$ or $_{3}^{+}$, or $\left._{2}^{-}\right\}$with or $_{3}^{+}=\{0,1\}^{3} \backslash$ $\{000\}$ and or $_{2}^{-}=\{0,1\}^{2} \backslash\{11\}$. Its NP-completeness follows from Schaefer's result [Sch78]. The problem can also be described as $\operatorname{CsP}(L)$, where $L$ is the clausal language $\{(+1,+1,+1),(-0,-0)\}$.

Problem: MONOTONE SAT

Input: A Boolean formula $\varphi=c_{1} \wedge \cdots \wedge c_{k}$ in conjunctive normal form, where each clause $c_{i}$ has either three positive literals or two negative literals.

Question: Is $\varphi$ satisfiable?

Proposition 14 If the 3-saturation $\widehat{L}$ contains a disjoint PN-pair of patterns then $\operatorname{CSP}(L)$ is $\mathrm{NP}$ complete.

Proof: Let $P=\left(+a_{1},+a_{2},+a_{3}\right)$ be a positive pattern of length 3 and $N=\left(-b_{1},-b_{2}\right)$ be the negative pattern of length 2 in $\widehat{L}$ that constitute the disjoint PN-pair. Let $d=\max (N)$. For each Boolean formula $\varphi$ we construct an $\widehat{L}$-formula $\varphi^{\prime}$ in the following way. For each clause or $_{3}^{+}(x, y, z)$ in $\varphi$ we put the clause $P(x, y, z)$ in $\varphi^{\prime}$ and for each clause ${ }^{\prime} r_{2}^{-}(x, y)$ in $\varphi$ we put the clause $N(x, y)$ in $\varphi^{\prime}$. Since $P$ and $N$ are disjoint it is easy to check that $\varphi$ is satisfiable if and only if $\varphi^{\prime}$ is (intuitively values smaller than or equal to $d$ will be identified to 0 , while the others will be identified to 1 ).

The proof for a positive pattern $P$ of length 2 and a negative pattern $N$ of length 3 follows from the previous case by the duality principle.

\section{Dichotomy Result}

We must analyze now the cases when the 3 -saturation $\widehat{L}$ does not contain a disjoint PN-pair. This will be done by a case analysis. 
Case 1: All positive patterns in $\widehat{L}$ have length 1, or dually all negative patterns in $\widehat{L}$ have length 1 .

This case corresponds to the Horn and dual Horn cases.

Proposition 15 Let $L$ be an $S U$-closed clausal language. If all positive (negative) patterns in $\widehat{L}$ have length 1 then both $L$ and $\widehat{L}$ are Horn (dual Horn).

Proof: Suppose that $L$ is not Horn, i.e., there exists a pattern $M$ with at least two positive literals. The clausal language $L$ is closed under subpatterns, therefore there must be a positive pattern $P=\left(+p_{1},+p_{2}\right)$ in $L$. Since $|P| \leq 3$ holds, we have that $P \in \widehat{L}$, what is a contradiction with the assumption. The result for dual Horn follows from the duality principle.

Tractability of the cases considered in Proposition 15 follows from Proposition 9.

Case 2: The 3-saturation $\widehat{L}$ contains at least one positive and one negative pattern of length greater or equal to 2 .

Case 2 splits to the following sub-cases.

Case 2.1: $\quad$ All patterns in $\widehat{L}$ have length smaller or equal to 2 .

If all patterns in $\widehat{L}$ have length smaller or equal to 2 then $L$ is bijunctive and $\operatorname{CsP}(L)$ is tractable by Proposition 9 .

Case 2.2: $\quad$ There exists at least one pattern $M \in \widehat{L}$ of length $|M|=3$.

We need to analyze the case when there exists a pattern $M \in \widehat{L}$ of length $|M|=3$. This is the most involved part of the proof. To this end we need to introduce the notions of $[a, b]$-valid, $[a, b]$-satisfiable, and $[a, b]$-unsatisfiable patterns on an interval $[a, b]$.

Definition 16 A pattern $M$ of length $k$ is called [a, $\boldsymbol{b}]$-valid if every assignment $I: V \rightarrow[a, b]$ satisfies the clause $M\left(x_{1}, \ldots, x_{k}\right)$, i.e., if every assignment whose range is restricted to the interval $[a, b]$ satisfies $M\left(x_{1}, \ldots, x_{k}\right) . M$ is called $[\boldsymbol{a}, \boldsymbol{b}]$-satisfiable if the clause $M\left(x_{1}, \ldots, x_{k}\right)$ is satisfiable by an assignment $I: V \rightarrow[a, b]$, i.e., by an assignment whose range is restricted to the interval $[a, b] . M$ is called $[\boldsymbol{a}, \boldsymbol{b}]$-unsatisfiable when there is no satisfying assignment $I: V \rightarrow[a, b]$ of the clause $M\left(x_{1}, \ldots, x_{k}\right)$, i.e., when there is no assignment restricted to the interval $[a, b]$ that satisfies $M\left(x_{1}, \ldots, x_{k}\right)$.

It is clear that on a given interval $[a, b]$ a pattern $M$ is $[a, b]$-valid, or $[a, b]$-satisfiable, or $[a, b]$ unsatisfiable. Moreover, $[a, b]$-validity implies $[a, b]$-satisfiability. The notion of $[a, b]$-satisfiability of $M$ means that we can restrict our attention to the satisfying assignments of $M\left(x_{1}, \ldots, x_{k}\right)$ on the interval $[a, b]$, contrary to the $[a, b]$-unsatisfiable patterns. It should also be clear that a pattern $M$ can be $[a, b]$-unsatisfiable even if the clause $M\left(x_{1}, \ldots, x_{k}\right)$ is satisfiable by an assignment $I$ whose range is not included in the interval $[a, b]$.

Definition 17 Let $L$ be a clausal language, such that $\widehat{L}$ contains a positive binary and a negative binary pattern. We call the values

$$
p_{\max }=\max \left\{\min (P) \mid P \in \operatorname{pos}_{2} \widehat{L}\right\} \quad \text { and } \quad q_{\min }=\min \left\{\max (N) \mid N \in \operatorname{neg}_{2} \widehat{L}\right\}
$$




$\begin{array}{lll}\text { Propagate L: } & (C \vee x \leq v) \wedge(x \leq u) \rightarrow(x \leq u) & \text { if } u \leq v \\ \text { Propagate R: } & (C \vee x \geq u) \wedge(x \geq v) \rightarrow(x \geq v) & \text { if } u \leq v \\ \text { Restrict L: } & (C \vee x \leq u) \wedge(x \geq v) \rightarrow C \wedge(x \geq v) & \text { if } u<v \\ \text { Restrict R: } & (C \vee x \geq v) \wedge(x \leq u) \rightarrow C \wedge(x \leq u) & \text { if } u<v \\ \text { Contradiction: } & (x \leq u) \wedge(x \geq v) \rightarrow \perp & \text { if } u<v\end{array}$

Figure 1: Formula simplification rules

the markers of the binary positive and negative patterns in the saturation $\widehat{L}$, respectively. We also call $P_{*}=\left(+p_{\max },+p_{\max }\right)$ and $N_{*}=\left(-q_{\min },-q_{\min }\right)$ the extremal patterns.

Observe that in Case 2.2 these markers are well-defined, since there are binary positive and negative patterns in the 3 -saturation $\widehat{L}$.

Our goal is to present a polynomial-time algorithm that decides the satisfiability of an $L$-formula $\varphi$. This algorithm starts with the following pre-processing step. From $\varphi$ we construct a new $L$ formula $\varphi^{\prime}$ by exhaustive application of the five rules presented in Figure 1. Since these rules are confluent and terminating, we can apply them in an arbitrary order and always obtain the unique normal form $\varphi^{\prime}$.

It is clear that $\varphi^{\prime}$ can be computed from $\varphi$ in polynomial time. The formulas $\varphi$ and $\varphi^{\prime}$ are logically equivalent, therefore they also have the same satisfiability property. Observe that every variable in $\varphi^{\prime}$ occurs at most once in a unary positive (resp. negative) clause. Moreover, if a variable $x$ occurs both in a positive clause $(x \geq u)$ and in a negative clause $(x \leq v)$, then these two clauses are compatible, i.e., $u \leq v$ holds, and both clauses are satisfied by two assignments $I(x)=u$ and $I^{\prime}(x)=v$. These observations will be used to justify the correctness of the polynomial-time algorithms developed in the sequel. Moreover, note that the patterns of all constraints occurring in $\varphi^{\prime}$ belong to $L$, since $L$ is closed under subpatterns.

Case 2.2 splits once more. We need to perform a case analysis on the position of markers of the binary monotone patterns in $\widehat{L}$.

Case 2.2.1: $\boldsymbol{p}_{\max } \leq \boldsymbol{q}_{\min }$. Observe that when the relation $p_{\max } \leq q_{\min }$ holds then there cannot be a disjoint $\mathrm{PN}$-pair of patterns in $L$.

Proposition 18 Let $L$ be an $S U$-closed clausal language, such that $\widehat{L}$ contains a positive binary, a negative binary, and a ternary pattern. If the markers satisfy $p_{\max } \leq q_{\min }$, then $\operatorname{CSP}(L)$ is decidable in polynomial time.

Proof: Let $\varphi$ be an $L$-formula. Transform $\varphi$ to a new $L$-formula $\varphi^{\prime}$ by an exhaustive application of the simplification rules from Figure 1. Observe that all non-unit clauses from $\varphi^{\prime}$ are $d$-valid for every $d \in\left[p_{\max }, q_{\min }\right]$. Indeed, for sake of contradiction, suppose that there exists a clause $c$ in $\varphi$, which is not $d$-valid for some $d \in\left[p_{\max }, q_{\min }\right]$. Let $M_{c}$ be the pattern associated to $c$. Since it is not $d$-valid, all literals of $M_{c}$ must be of the form $+p$ with $p>d \geq p_{\max }$ or $-q$ with $q<d \leq q_{\min }$. $M_{c}$ cannot be positive since otherwise we can produce from $M_{c}$ by taking subpatterns a positive binary pattern $P=\left(+p_{1},+p_{2}\right) \in \widehat{L}$ with $p_{i}>p_{\max }$ for each $i$, but this contradicts the definition of $p_{\max }$ and the fact that the SU-closed clausal language $L$ is closed under subpatterns. Dually, $M_{c}$ cannot be negative since otherwise we could produce a negative binary pattern $N=\left(-q_{1},-q_{2}\right) \in \widehat{L}$ with $q_{i}<p_{\max } \leq q_{\min }$, which contradicts the definition of $q_{\min }$ and the SU-closedness of $L$. If $M_{c}$ is 
mixed, we can derive by taking subpatterns a pattern $M^{\prime}=(+p,-q) \in \widehat{L}$ with $q<p_{\max }<p$. There exists by definition a pattern $P_{1}=\left(+p_{\max },+p_{1}\right) \in \widehat{L}$ for some $p_{1} \geq p_{\max }$. We obtain by two resolution steps from $M^{\prime}$ and $P_{1}$ the pattern $Q=(+p,+p) \in \widehat{L}$, which contradicts the definition of $p_{\max }$, since $p>p_{\max }$ holds.

After the transformation to $\varphi^{\prime}$ by an exhaustive application of the rules from Figure 1, the algorithm to find a satisfying assignment proceeds as follows. If $\varphi^{\prime}$ contains the empty clause $\perp$ then $\varphi$ is unsatisfiable. Otherwise, assign first all variables to an arbitrary but fixed $d \in\left[p_{\max }, q_{\min }\right]$. This initial assignment $I(x)=d$ for all $x \in V$ satisfies all non-unit clauses, according to the previous observation, but not necessarily the unit ones. If a variable $x$ appears in a unit clause $(x \geq a)$ (or $x \leq b)$ not satisfied by $I(x)=d$, then change the value of $x$ to $a$ (resp. $b$ ).

We need to prove that these assignment modifications do not alter the satisfaction of the nonunit clauses. Suppose that $x$ occurs in $\varphi^{\prime}$ in a unit positive clause $(x \geq v)$ which is not not satisfied by $I(x)=d$, i.e., we have $v>d$. There are three types of clauses in $\varphi^{\prime}$ where the variable $x$ can occur, namely a negative unit clause $(x \leq u)$, as well as the non-unit clauses $(C \vee x \geq u)$ with $u>v$ and $(C \vee x \leq u)$ with $u \geq v$. A negative unit clause $(x \leq u)$ must be compatible with $(x \geq v)$, since $\varphi^{\prime}$ does not contain the empty clause, thus it is satisfied by $I(x)=v$. The assignment $I(x)=d$ does not contribute to the satisfaction of the clause $(C \vee x \geq u)$ since $d<v \leq u$ holds. The asignment $I(x)=d$ satisfies the clause $(C \vee x \leq u)$, since $d<v \leq u$ holds, thus $I(x)=v$ satisfies it, too. Therefore changing the assignment from $I(x)=d$ to $I(x)=v$ will not affect the satisfiability of the non-unit clauses.

Case 2.2.2: $\boldsymbol{q}_{\min }<\boldsymbol{p}_{\text {max }}$. In this case the $\operatorname{CSP}(L)$ problem can be substantially simplified and studied on the restricted interval $\left[q_{\min }, p_{\max }\right]$.

Lemma 19 Let $L$ be an $S U$-closed clausal language such that $\widehat{L}$ contains a positive and a negative binary pattern. If the markers satisfy the condition $q_{\min }<p_{\max }$ then both extremal patterns $N_{*}=$ $\left(-q_{\min },-q_{\min }\right)$ and $P_{*}=\left(+p_{\max },+p_{\max }\right)$ belong to $\widehat{L}$.

Proof: By definition of the markers $q_{\min }$ and $p_{\max }$, there exist patterns $N=\left(-q_{1},-q_{\min }\right) \in \widehat{L}$ and $P=\left(+p_{1},+p_{\max }\right) \in \widehat{L}$, where $q_{1} \leq q_{\min }<p_{\max } \leq p_{1}$ holds. A resolution step between $P$ and $N$ produces the pattern $M=\left(-q_{\min },+p_{\max }\right)$. Another resolution step between $M$ and $N$ produces $N_{*}$, whereas a different resolution step between $M$ and $P$ produces $P_{*}$.

Lemma 20 Let $L$ be an SU-closed clausal language such that $\widehat{L}$ contains a positive binary, a negative binary, and a ternary pattern. If $\widehat{L}$ does not contain a disjoint $P N$-pair and the markers satisfy the condition $q_{\min }<p_{\max }$, then every pattern $M \in L$ of length $|M| \geq 3$ is $\left[q_{\min }, p_{\max }\right]$-valid.

Proof: Observe first that both extremal patterns $N_{*}=\left(-q_{\min },-q_{\min }\right)$ and $P_{*}=\left(+p_{\max },+p_{\max }\right)$ belong to $\widehat{L}$, according to Lemma 19. Suppose that there exists a pattern $M \in L$ of length $|M| \geq 3$, which is not $\left[q_{\min }, p_{\max }\right]$-valid. This means that $M$ contains only the literals $-b$ with $b<p_{\max }$ and $+a$ with $a>q_{\min }$.

Let $M$ be positive, i.e., $M=\left(+a_{1}, \ldots,+a_{k}\right)$, where $k \geq 3$ and $a_{i}>q_{\text {min }}$ holds for all $i$. Then the subpattern $M_{3}^{+}=\left(+a_{1},+a_{2},+a_{3}\right)$ belongs to $\widehat{L}$, and together with $N_{*}=\left(-q_{\min },-q_{\min }\right)$ forms a disjoint $\mathrm{PN}$-pair, which is a contradiction with the assumption. Let $M$ be negative, i.e., $M=\left(-b_{1}, \ldots,-b_{k}\right)$, where $k \geq 3$ and $b_{i}<p_{\max }$ holds for all $i$. Then, the subpattern $M_{3}^{-}=$ $\left(-b_{1},-b_{2},-b_{3}\right)$ belongs to $\widehat{L}$, and together with $P_{*}=\left(+p_{\max },+p_{\max }\right)$ forms a disjoint PN-pair, 
which is once more a contradiction with the assumption. Let $M=\left(+a_{1}, \ldots,+a_{k},-b_{1}, \ldots,-b_{\ell}\right)$ be mixed, where $k+\ell \geq 3, a_{i}>q_{\min }$, and $b_{j}<p_{\max }$ hold for all $i, j$. Then either the subpattern $M_{3}=\left(+a_{1},+a_{2},-b_{1}\right)$ or the subpattern $M_{3}=\left(+a_{1},-b_{1},-b_{2}\right)$ belongs to $\widehat{L}$. Thus, by repeated resolution of $M_{3}$ and $P_{*}$ we can produce the positive pattern $M_{3}^{\prime}=\left(+a_{1},+p_{\max },+p_{\max }\right)$, where $a_{1}>q_{\min }$ and $p_{\max }>q_{\min }$ hold. This leads to a contradiction as before.

Lemma 21 Let $L$ be an SU-closed clausal language such that $\widehat{L}$ contains a positive binary, a negative binary, and a ternary pattern. If $\widehat{L}$ does not contain a disjoint $P N$-pair and the markers satisfy the condition $q_{\min }<p_{\max }$, then every binary pattern in $L$ is either $\left[q_{\min }, p_{\max }\right]$-valid or of the form $(+a,+b),(-a,-b)$, or $(-a,+b)$, where $a, b \in\left[q_{\min }, p_{\max }\right]$.

Proof: Recall that both extremal patterns $N_{*}=\left(-q_{\min },-q_{\min }\right)$ and $P_{*}=\left(+p_{\max },+p_{\max }\right)$ belong to $\widehat{L}$, according to Lemma 19 .

Let $P=(+a,+b) \in L$ be a positive pattern with $a \leq b$. $P$ belongs to $\widehat{L}$. If $a \leq q_{\min }$ holds then $P$ is $\left[q_{\min }, p_{\max }\right]$-valid. Consider now the case $q_{\min }<a$. The pattern $Q_{*}=\left(-q_{\min },+p_{\max }\right)$ is a resolvent of the extremal patterns $P_{*}=\left(+p_{\max },+p_{\max }\right)$ and $N_{*}=\left(-q_{\min },-q_{\min }\right)$ since $q_{\min }<p_{\max }$ holds. If $q_{\min }<a \leq p_{\max }$ holds then we obtain $Q^{\prime}=\left(+b,+p_{\max }\right)$ by resolution from $P$ and $Q_{*}$. By resolution from $Q^{\prime}$ and $N_{*}$ we get $Q^{\prime \prime}=\left(+b,-q_{\text {min }}\right)$. Another resolution step between $Q^{\prime}$ and $Q^{\prime \prime}$ gives $Q_{\dagger}=(+b,+b)$. If $b>p_{\max }$ then this contradicts the definition of $p_{\max }$, therefore we must have $b \leq p_{\max }$ and $[a, b] \subseteq\left[q_{\min }, p_{\max }\right]$. The same result holds by duality for negative binary patterns in $L$.

Let $M=(-a,+b) \in L$. If $a \geq p_{\max }$ or $b \leq q_{\min }$ hold then $M$ is $\left[q_{\min }, p_{\max }\right]$-valid. If $a<p_{\max }$ and $b>q_{\min }$ hold then we need a supplementary case analysis. If $a \geq q_{\min }$ and $b \leq p_{\max }$ then the interval $[a, b]$ or $[b, a]$, depending whether $a<b$ or $b \leq a$, is included in $\left[q_{\min }, p_{\max }\right]$. If $a<q_{\min }$ then we can produce by resolution from $M$ and $N_{*}=\left(-q_{\min },-q_{\min }\right)$ the pattern $Q=\left(-a,-q_{\min }\right)$, followed by an additional resolution step with $M$ which gives $Q_{\dagger}=(-a,-a)$, what contradicts the definition of $q_{\min }$. If $b>p_{\max }$ holds then we can produce by resolution from $M$ and $P_{*}=\left(+p_{\max },+p_{\max }\right)$ the pattern $Q^{\prime}=\left(+p_{\max },+b\right)$, followed by two additional resolution steps as above, which give $Q_{\dagger}=(+b,+b)$, what contradicts the definition of $p_{\max }$.

Proposition 22 Let $L$ be an $S U$-closed clausal language such that $\widehat{L}$ contains a positive binary, a negative binary, and a ternary pattern. If $\widehat{L}$ does not contain a disjoint $P N$-pair and the markers satisfy the condition $q_{\min }<p_{\max }$, then $\operatorname{CsP}(L)$ is decidable in polynomial time.

Proof: The algorithm to find a satisfying assignment for $\varphi$ in polynomial time proceeds as follows. First, it transforms $\varphi$ to $\varphi^{\prime}$ by an exhaustive application of the rules from Figure 1. If $\varphi^{\prime}$ contains the empty clause $\perp$ then $\varphi$ is unsatisfiable. Otherwise, construct the sub-formula $\varphi_{2}^{\prime}$ consisting of all $\left[q_{\min }, p_{\max }\right]$-satisfiable clauses from $\varphi^{\prime}$ which are not $\left[q_{\min }, p_{\max }\right]$-valid. According to Lemmas 20 and 21, $\varphi_{2}^{\prime}$ is bijunctive, i.e., it contains only unit and binary clauses, and therefore its satisfiability is decidable in polynomial time. Find a satisfying assignment for $\varphi_{2}^{\prime}$. The binary clauses from formula $\varphi^{\prime}$ involve only comparisons with values from the interval $\left[q_{\min }, p_{\max }\right]$, according to Lemma 21. Hence, if we change any assignment $I(x)<q_{\min }$ to $I(x)=q_{\min }$ and any assignment $I(x)>p_{\max }$ to $I(x)=p_{\max }$, this cannot affect the truth value of any literal in $\varphi_{2}^{\prime}$. Therefore if $\varphi_{2}^{\prime}$ is satisfiable, it must be $\left[q_{\min }, p_{\max }\right]$-satisfiable. 
The remaining clauses in $\varphi^{\prime}$ are either "big" clauses, i.e., of length 3 or more, binary clauses, or unit clauses. The first two types are $\left[q_{\min }, p_{\text {max }}\right]$-valid, so the computed satisfying assignment satisfies them. What remains are unit clauses which are not $\left[q_{\min }, p_{\max }\right]$-valid.

As in the proof of Proposition 18, we change the values of the concerned variables, setting $I(x)=v$ if either $x \leq v$ or $x \geq v$ is a $\left[q_{\min }, p_{\max }\right]$-unsatisfiable clause in $\varphi^{\prime}$. Once more, by construction of $\varphi^{\prime}$ for the same reason as in the proof of Proposition 18, this change does not alter the satisfaction of the already satisfied clauses. Suppose that $x$ occurs in $\varphi^{\prime}$ in a unit positive clause $(x \geq v)$ which is not satisfied by $I(x)=d$, i.e., we have $v>d$. There are three types of clauses in $\varphi^{\prime}$ where the variable $x$ can occur, namely a negative unit clause $(x \leq u)$, as well as the non-unit clauses $(C \vee x \geq u)$ with $u>v$ and $(C \vee x \leq u)$ with $u \geq v$. A negative unit clause $(x \leq u)$ must be compatible with $(x \geq v)$, since $\varphi^{\prime}$ does not contain the empty clause, thus it is satisfied by $I(x)=v$. The assignment $I(x)=d$ does not contribute to the satisfaction of the clause $(C \vee x \geq u)$ since $d<v \leq u$ holds. The asignment $I(x)=d$ satisfies the clause $(C \vee x \leq u)$, since $d<v \leq u$ holds, thus $I(x)=v$ satisfies it, too. Therefore changing the assignment from $I(x)=d$ to $I(x)=v$ will not affect the satisfiability of the non-unit clauses.

To the best of our knowledge, the tractable cases identified by means of Proposition 22 are new. The following theorem follows from Propositions 14, 15, 18, and 22.

Theorem 23 (Dichotomy Theorem) Let L be an SU-closed clausal language. If the saturation $\widehat{L}$ does not contain a disjoint PN-pair then $\operatorname{CSP}(L)$ is decidable in polynomial time, otherwise $\operatorname{CSP}(L)$ is NP-complete.

Example 24 Consider the SU-closed clausal language

$$
\begin{aligned}
L= & \{(-0),(-1),(+1),(+2), \\
& (+1,-0),(+1,+1),(-1,-1),(+2,+2), \\
& (+1,+1,+1)\}
\end{aligned}
$$

over the 3 -element domain $D=\{0,1,2\}$. The only endomorphism of $L$ is the identity. The 3 saturation $\widehat{L}$ of $L$ is then equal to $L \cup\{(+2,-1),(+1,+2),(+1,-1)\}$. There is no disjoint PN-pair in $\widehat{L}$, therefore $\operatorname{CSP}(L)$ is polynomial-time decidable.

As usual, a clausal language with a polynomial-time decidable problem $\operatorname{CSP}(L)$ is called tractable. The following theorem proves that we can recognize tractable clausal languages in polynomial time, giving the answer to the meta-problem of Theorem 23.

Theorem 25 Let $L$ be an arbitrary clausal language over a fixed domain D. It is decidable in polynomial time whether $\operatorname{CSP}(L)$ is tractable.

Proof: Starting with a clausal language $L$, we can get rid of the redundant values, according to Lemma 3 and produce the corresponding SU-closed language $L_{1}$, according to Proposition 7 , both in polynomial time with respect to the size of $L$. From $L_{1}$ we can produce the 3 -saturation $\widehat{L}_{1}$ in linear time with respect to the size of $L_{1}$, according to Remark 11 . The 3 -saturation can be searched in polynomial time for a PN-pair. 


\section{Concluding Remarks}

We presented a complexity analysis of the constraint satisfaction problems over finite totally ordered domains, based on the new concept of clausal patterns. We derived decidable conditions for CSP problems implying either NP-completeness or polynomial-time satisfiability. In fact, our polynomial-time cases generalize the previously known characterization through Horn, dual Horn, bijunctive, 0- and 1-valid Boolean relations. Not surprisingly the 3-saturation concept allows us to decide algorithmically the dividing condition between the tractable and intractable instances. The result is a dichotomy theorem applicable to the CSP problems over totally ordered domains of arbitrary cardinality.

By combining tractability results from the paper with the algebraic approach, it is possible to obtain many more tractable (non-clausal) CSPs. We leave this as an open problem - to characterize tractable (non-clausal) constraint languages that can be generated from clausal ones. This would actually amount to describing tractable clausal languages algebraically. Our CSP problems are somewhat orthogonal to the relationally defined ones. On one hand we obtained a dichotomy theorem, on the other hand relations can express much more constraints than clauses, so our classification is coarser. Another possible extension of our research is to consider clausal patterns over a partially rather than totally ordered domain. We believe that the results presented in this paper can be generalized to this more general case.

Acknowledgment: We sincerely thank Julien Demouth for proofreading the paper and pointing out several writing errors. We also thank the anonymous referee for his useful remarks.

\section{References}

[BFS01] M. Baaz, Ch. G. Fermüller, and G. Salzer. Automated deduction for many-valued logics. In J. A. Robinson and A. Voronkov, editors, Handbook of Automated Reasoning, volume 2, chapter 20, pages 1355-1402. Elsevier Science, 2001.

[BJK99] A. Bulatov, P. Jeavons, and A. Krokhin. Constraint satisfaction problems and finite algebras. Technical Report TR-4-99, Oxford University Computing Laboratory, 1999.

[BJK05] A. Bulatov, P. Jeavons, and A. Krokhin. Classifying the complexity of constraints using finite algebras. SIAM Journal on Computing, 34(3):720-742, 2005.

[Bul02] A. A. Bulatov. A dichotomy theorem for constraints on a three-element set. In Proceedings 43rd Symposium on Foundations of Computer Science (FOCS 2002), Vancouver (British Columbia, Canada), pages 649-658, November 2002.

[Bul03] A. A. Bulatov. Tractable conservative constraint satisfaction problems. In Proceedings 18th IEEE Symposium on Logic in Computer Science (LICS 2003), Ottawa (Canada), pages 321-330, June 2003.

[CJJK00] D. Cohen, P. Jeavons, P. Jonsson, and M. Koubarakis. Building tractable disjunctive constraints. Journal of the Association for Computing Machinery, 47(5):826-853, 2000. 
[CKS01] N. Creignou, S. Khanna, and M. Sudan. Complexity Classifications of Boolean Constraint Satisfaction Problems, volume 7 of SIAM Monographs on Discrete Mathematics and Applications. SIAM, Philadelphia (PA), 2001.

[Dal02] V. Dalmau. Constraint satisfaction problems in non-deterministic logarithmic space. In P. Widmayer, F. Triguero Ruiz, R. Morales Bueno, M. Hennessy, S. Eidenbenz, and R. Conejo, editors, Proceedings 29th International Conference on Automata, Languages, and Programming (ICALP 2002), Malaga (Spain), volume 2380 of Lecture Notes in Computer Science, pages 414-425. Springer-Verlag, July 2002.

[FV98] T. Feder and M. Y. Vardi. The computational structure of monotone monadic SNP and constraint satisfaction: a study through Datalog and group theory. SIAM Journal on Computing, 28(1):57-104, 1998.

[GHSZ04] A. Gil, M. Hermann, G. Salzer, and B. Zanuttini. Efficient algorithms for constraint description problems over finite totally ordered domains. In D. Basin and M. Rusinowitch, editors, Proceedings 2nd International Joint Conference on Automated Reasoning (IJCAR'04). Cork (Ireland), volume 3097 of Lecture Notes in Computer Science, pages 244-258. Springer-Verlag, July 2004.

[Häh01] R. Hähnle. Complexity of many-valued logics. In Proceedings 31st IEEE International Symposium on Multiple-Valued Logic (ISMVL 2001), Warsaw (Poland), pages 137-148. IEEE Computer Society, May 2001.

[HN04] P. Hell and J. Nešetřil. Graphs and Homomorphisms. Oxford University Press, 2004.

[JC95] P. Jeavons and M. C. Cooper. Tractable constraints on ordered domains. Artificial Intelligence, 79(2):327-339, 1995.

[KV00] P. G. Kolaitis and M. Y. Vardi. Conjunctive-query containment and constraint satisfaction. Journal of Computer and System Science, 61(2):302-332, 2000.

[Lad75] R. E. Ladner. On the structure of polynomial time reducibility. Journal of the Association for Computing Machinery, 22(1):155-171, 1975.

[Sch78] T. J. Schaefer. The complexity of satisfiability problems. In Proceedings 10th Symposium on Theory of Computing (STOC'78), San Diego (California, USA), pages 216-226, 1978. 